\title{
A Control and Monitoring Oriented Model of a Film Manufacturing Process *
}

\author{
Sung-ho Hur* Jonas Balderud* Reza Katebi * \\ Andrew Taylor ${ }^{* *}$ \\ * Industrial Control Centre, University of Strathclyde, UK (e-mail: \\ j.balderud@eee.strath.ac.uk). \\ ** DuPont Teijin Films UK Ltd
}

\begin{abstract}
This paper describes the development of a control and monitoring oriented model of a plastic film manufacturing process. The model is mainly derived from first-principles and has been implemented in the Matlab/Simulink dynamic simulation environment.

The development of the model forms the first phase of a project that aims to develop a nonlinear sub-space based monitoring, fault detection and trouble shooting system for the film manufacturing process.
\end{abstract}

Keywords: Dynamic Modeling, Film Manufacturing, Monitoring

\section{INTRODUCTION}

This paper focuses on the development of a monitoring and control oriented model of a film manufacturing process that produces thin plastic film from polymer. The development of this model forms the first phase of a project that aims to develop a nonlinear sub-space based monitoring, fault detection and trouble shooting system that will aid in the operation and control of the film process.

The film manufacturing process considered in this work starts with polymer heated to above its melting point such that it can be treated as a viscous fluid. Using a continuous casting process the polymer melt is formed into a thick film that is approximately 1.5 meter wide in one direction and continuous in the other. This film is then mechanically stretched in both directions such that a much thinner film is obtained. The stretching operations are carried out under high temperatures and in a continuous manner which produces a film that can be wound onto a large roll.

From a control perspective, the challenge is to ensure that the thickness profile of the finished film remains uniform, both in the machine direction and the cross-machine direction Featherstone et al. [2000]. This typically led to a large scale multivariable control problem that is difficult to manage due to its shear size. An additional complicating fact is that there is typically a significant transport delay between actuators and sensors, which reduces the performance achievable by feedback control. In order to tackle these difficulties model-based feedforward control schemes are often employed Corscadden and Duncan [2000], Duncan et al. [2000].

Due to the past and present interest in model-based control techniques, there already exist a vast range of control

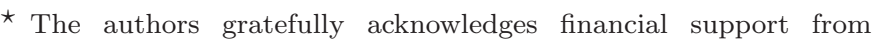
Dupont Teijin Films Ltd, UK, EPSRC Case Award, UK, and EPSRC platform Grant (GR/R04683/01), UK.
}

oriented models. However, since these models are predominately empirical their suitability in fault-detection and trouble shooting applications, where model-plant mapping is required, are limited. The model developed in this paper, by contrast, breaks with tradition and is largely developed from first principles, taking account of mass transfer, heat transfer and deformation characteristics of the unit operations of the film manufacturing process.

This paper is organised as follows: The film manufacturing process is described in section 2. A mathematical model of the film process is described in section 3 , which is followed by model tuning in section 4 and model validation in section 5. Conclusions are presented in section 6 .

\section{PROCESS DESCRIPTION}

Figure 1 depicts the process flow diagram of the film manufacturing process considered in this work. The film manufacturing process starts with a polymer melt that is fed into the die component of the film process. Inside the die the polymer melt is distributed along the crossmachine direction and discharged through the die gap onto a rotating casting drum, whereby a thick and continuous polymer film is formed on the surface of the drum. By continuously cooling the casting drum using cold water the polymer melt quickly solidifies. The polymer film is then transported along the surfaces of the casting drum, quench roll and slow-nip rolls and eventually reaches the pre-heat rolls.

The purpose of the pre-heat rolls is to raise the temperature of the film prior to it being stretched in the forward draw. The pre-heat rolls are therefore heated by hot water, which raises the temperature of the film to approximately $90{ }^{\circ} \mathrm{C}$ at the end of the pre-heat roll section. A speed differential between the pre-heat rolls and cooling rolls is then used to stretch the film to approximately 3 times its original length in the the machine direction. To set the dimensions of the stretched film, the film needs to be 


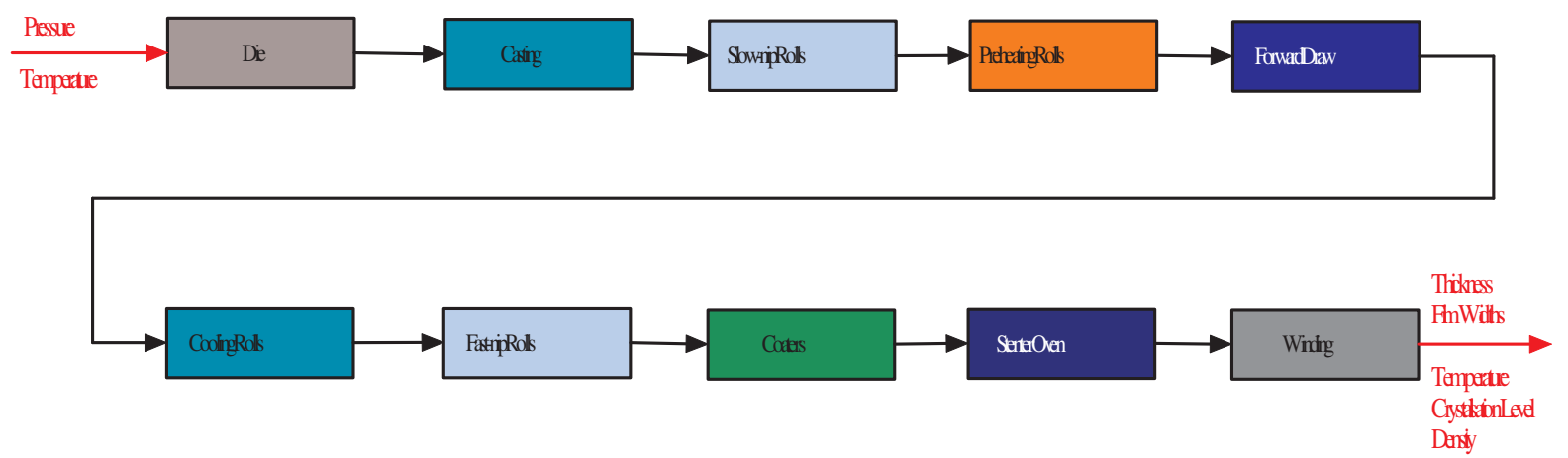

Fig. 1. The Process

quickly cooled down, which is carried out by the cooling rolls. After leaving the cooling roll section the film travels through the fast-nip roll section and through the coater where a small amount of coating material and water is deposited to the surface of the film.

The stenter oven can be divided into four main sections: the pre-heat oven, the sideways draw, the crystalliser and the cooling zone. As the film enters the stenter oven, the clips grip the edges of the film. These clips move in the machine direction at the same speed, running on a track that extends the length of the stenter. The film temperature is raised towards the point where it can be drawn in the pre-heat oven using heated air. In the sideways draw oven the distance between the tracks on which the clips run gradually increases, thus causing the film to stretch in the cross-machine direction. Typically the width of the film is increased in the sideways draw to 3 times its original width. The stretched film structure is then heat set in the crystalliser and cooling zone by first heating the film and then quickly cooling the film. The finished film, which have been stretched 3 times in the machine direction and 3 times in the cross-machine direction and therefore is approximately 9 times thinner than its original thickness is finally spooled onto a roll and eventually transported away for post processing.

In order to control the machine direction and crossmachine direction thickness profiles of the finished film the thickness profile of the film formed on the casting drum is varied. By adjusting the mass flow rate discharged through the die gap the film thickness on the casting drum can be adjusted. To enable fine tuning of the mass flow rate at positions along the width of the die gap the die is equipped with a large number of die bolt heaters that locally heat the polymer melt which decreases the melt viscosity and thus increases the mass flow rate.

\section{MATHEMATICAL MODEL}

The main modelling objective is to track changes in the film thickness profile throughout the film line process. However, in order to achieve this it is necessary to track the temperature of the film (or polymer melt) and the velocity of the film at positions along the machine direction and cross-machine direction of the film line process.

For modelling purposes the film line process is divided into a series of unit operations. The decomposition into unit operations follows the structure of the process in
Figure 1, giving the following set of operations: die, casting (sheet forming and cooling), transport along the slownip rolls, heating and transport through the pre-heat rolls, forward draw, cooling and transport through the cooling rolls, transport through the fast-nip rolls, coating, heating and transport in the pre-heat oven, sideways draw and heating, cooling and transport in the crystalliser and cooling zone. Within each of these unit operations the film path is meshed in the machine ( $x$-direction) and cross-machine ( $y$-direction) directions using a uniform rectangular mesh. Sub-models for mass-transfer, heattransfer and deformation are then formulated for each unit operation and fitted to the mesh.

In the next two sub-sections, the mass and heat transfer sub-models are explained, which are common for all unit operations. These sub-sections are then followed by a set of sub-sections that explain how these generic models are applied to each of the unit operations.

\subsection{Mass transfer model}

In order to capture dynamic variations in film thickness, the mass of the film at positions along the machine and cross-machine directions of the film path must be tracked. Assuming that the film in travels at velocity, $v_{m}(t)(\mathrm{m} / \mathrm{s})$, in the machine direction of the film line process, then the mass at position $(i \Delta x, j \Delta y)$ along the length and width of the film line, where $\Delta x$ and $\Delta y$ is the length and width of each mesh section, is governed by the following differential equation,

$$
\frac{\partial W_{i, j}(t)}{\partial t}=v_{m}(t) \frac{W_{i-1, j}(t)-W_{i, j}(t)}{\Delta x}
$$

where $W_{i, j}(t)$ denotes the mass (in $\mathrm{kg}$ ) per unit of area (in $\mathrm{m}^{2}$ ). The above equation governs the mass flow in each of the rectangular mesh sections along the film path. Note that in the above it is assumed that the film velocity remains constant. This is not the case in the forward draw, where the film is stretched in the machine direction and a different mass transfer equation is required.

\subsection{Heat transfer model}

Due to the relatively high transportation velocity of the film, it is assumed that conductive heat transfer within the film can be ignored. The temperature, $T_{i, j}(t)$, at position 


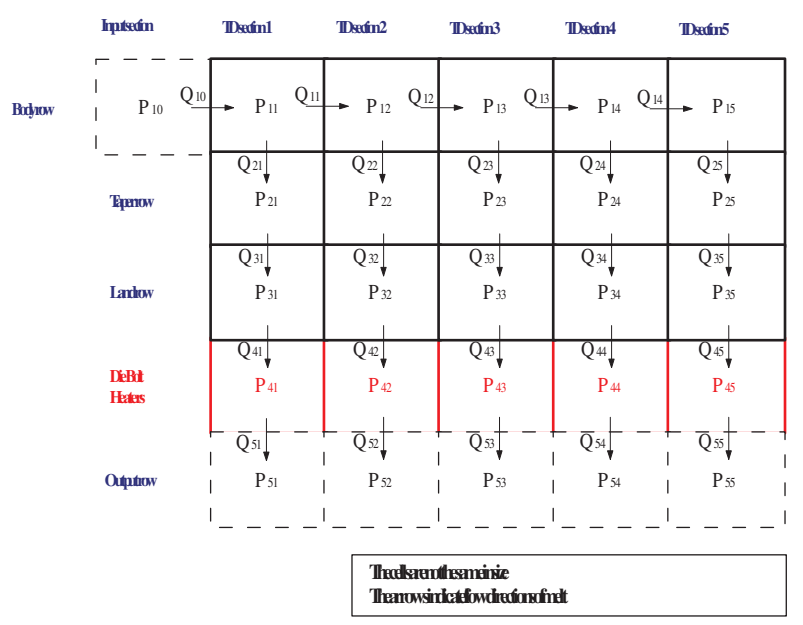

Fig. 2. 2-D explanatory view of the die component of the film manufacturing process

$(i \Delta x, j \Delta y)$ can therefore be modeled using the following convective heat transfer equation,

$$
\frac{\partial T_{i, j}(t)}{\partial t}=v_{m}(t) \frac{T_{i-1, j}(t)-T_{i, j}(t)}{\Delta x}+\frac{Q(t)}{c_{f} W_{i, j}(t) \Delta x \Delta y}
$$

where $c_{f}$ denote the specific heat of the film (in $\mathrm{J} / \mathrm{kg} / \mathrm{K}$ ) and where $Q(t)$ denote the heat added or removed from the film (in $\mathrm{J} / \mathrm{s}$ ).

The top and bottom surface of the film is either in contact with a roller surface or exposed to air. For the surface that is in contact with a roller surface the following heat flow equation is used,

$$
Q_{c}(t)=\Delta x \Delta y h_{c}\left(T_{c}(t)-T_{i, j}(t)\right)
$$

where $h_{c}$ denote the heat transfer coefficient (in $\mathrm{J} / \mathrm{s} / \mathrm{m}^{2} / \mathrm{K}$ ) and where $T_{c}(t)$ denote the temperature of the cooling/heating medium inside the roller. For the film surface exposed to the air heat transfer by radiation and convection is considered, leading to the following heat transfer equation,

$$
\begin{aligned}
Q_{a}(t)= & \Delta x \Delta y\left(h_{a}\left(T_{a}(t)-T_{i, j}(t)\right)\right. \\
& +\Delta x \Delta y\left(\sigma \epsilon\left(T_{a}(t)^{4}-T_{i, j}(t)^{4}\right)\right)
\end{aligned}
$$

where $\sigma$ denote Stefan Boltzmann's constant (in $\mathrm{J} / \mathrm{s} / \mathrm{m}^{2} / \mathrm{K}^{4}$ ) and where $\epsilon$ denotes the emissivity.

Heat transfer within the stenter is mainly carried out by blowing hot or cold air onto the surface of the film, in which case only the first term of Equation 4 is employed with an appropriate heat transfer constant that takes into account of the air velocity.

\subsection{Die}

The die component of the film line process is fed by polymer melt. This melt enters the die through a circular pipe connected to one of the sides of the die. Due to the mechanical design of the die the melt flow is distributed approximately evenly along the width of the die and subsequently discharged through the die gap onto the casting drum. Die bolt heaters mounted across the width of the die are used by the cross-directional control system to manipulate the melt flow. The actuator outputs for these heaters are provided by the cross-directional control system based on the measured finished film thickness profile deviation from target.

For modelling purposes the die is viewed as being comprised of a set of interconnected pipes that forms a flow mesh as shown in Figure 2. The flow rate between each of the rectangular sections shown in Figure 2 is governed by Poiseuille's law for laminar flow of a viscous fluid,

$$
P_{i+1, j}-P_{i, j}=\frac{8 \mu L_{i, j}}{\pi R_{i, j}^{4}} Q_{i+1, j}
$$

and,

$$
P_{i, j+1}-P_{i, j}=\frac{8 \mu L_{i, j}}{\pi R_{i, j}^{4}} Q_{i, j+1}
$$

where $\mu$ is the viscosity of the polymer melt, $L_{i, j}$ and $R_{i, j}$ is the equivalent pipe length and radius of each flow section, $P_{i, j}$ is the pressure in section $(i, j)$ and $Q_{i, j}$ is the volumetric flow rate in section $(i, j)$.

To model the effect of the die bolt heaters the following pressure flow relation is used,

$$
P_{i+1, j}-P_{i, j}=k_{j} Q(i, j)
$$

where the coefficient $k_{j}$ is a friction factor given by,

$$
k_{j}=\beta_{j} x_{j}+\sum_{n=1}^{N_{s}}\left(\beta_{j} x_{j-n}+\beta_{j} x_{j+n}\right)
$$

where $\beta_{j}$ are scalar coefficients and $x_{j}$ are die bolt heater settings. The form of (8) is derived from the observation that the heating setting of a particular die bolt heater affect the flow rate of in a neighbourhood of the die bolt heater.

By combining the above equations with appropriately selected continuity equations the flow conditions within the die can be computed by solving a linear system of equations.

\subsection{Casting Drum}

When the polymer melt is discharged through the die gap onto the surface of the casting drum, a thick film is formed. The thickness of this film is determined by the mass flow rate leaving the die gap and the speed of the casting drum. In order to compute the mass per unit of area deposited onto the casting drum at a position $j \Delta y$ along the width of the casting drum the following formula is used:

$$
W_{j}(t)=\frac{\dot{m}_{j}(t)}{v_{m}(t) \Delta y_{j}\left(v_{m}(t), T_{j}(t)\right)}
$$

where $\dot{m}_{j}(t)$ denote the mass flow rate (in $\mathrm{kg} / \mathrm{s}$ ) discharged through the die gap at position $j \Delta y, v_{m}(t)$ denote the velocity of the casting drum and where $\Delta y_{j}\left(v_{m}(t), T_{j}(t)\right)$ refer to a scalar function that returns correction factor to compensate for the fact that the film shrinks in the width direction near the edges. The function $\Delta y_{j}\left(v_{m}(t), T_{j}(t)\right)$ may be obtained by solving a computational fluid dynamics problem for different casting drum velocities and discharge temperatures. 


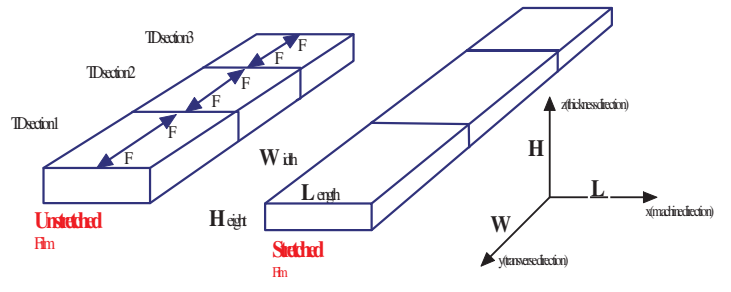

Fig. 3. Sideways Draw

The heat transfer and mass transfer associated with the casting drum process is modeled using the heat and mass transfer models shown in Section 3.1 and 3.2.

\subsection{Pre-heating, Slow-nip, Fast-nip, and Cooling Rolls}

These unit operations are all modeled using the previously presented mass and heat transfer models.

\subsection{Forward draw}

During the forward draw process the film is stretched in the machine direction. This is facilitated by employing a speed differential between the slow-nip and fast-nip rolls. The mass flow rate leaving the slow-nip rolls at position $j \Delta y$ can be computed from,

$$
\dot{m}_{j}(t)=W_{j}(t) v_{s}(t) \Delta y
$$

where $v_{s}(t)$ denote the velocity of slow nip rolls. Due to conservation of mass the mass flow rate at the fast nip rolls must be the same, giving the following relation to compute the stretched weight of the film, $\tilde{W}_{j}(t)$,

$$
\tilde{W}_{j}(t)=\frac{W_{j}(t) \Delta y v_{s}(t)}{v_{f}(t) \Delta y_{j}\left(v_{f}(t) / v_{s}(t), T_{j}(t)\right)}
$$

where $v_{f}(t)$ denote the velocity of fast nip rolls and where $\Delta y_{j}\left(v_{f}(t) / v_{s}(t), T_{j}(t)\right)$ refer to a scalar nonlinear function that compensates for non-uniform stretching in the crossmachine direction.

\subsection{Coaters}

The coater is modeled using the generic equations for heat and mass transfer shown in Section 3.1 and 3.2.

\subsection{Stenter Oven}

The stenter oven is comprised of the pre-heat oven, the sideways draw, the crystalliser and the cooling zone. Apart from the sideways draw these unit operations can be modeled using the generic models for heat and mass transfer previously presented.

Sideways Draw During the sideways draw the film is stretched in the cross-web direction. The behaviour of the film during stretching depends on many factors, including how fast the film is stretched, the temperature of the film and the thickness of the film.

In order to develop a mathematical model of the sideways draw process a cross-machine directional strip of the film will be considered, as shown in Figure 3. For simplicity the film strip shown in Figure 3 have only been divided into three cross-machine directional sections. This number is higher in reality.

Figure 3 illustrates the effect of stretching. It is important to observe that the un-stretched film is divided into sections with uniform widths, but different heights. The stretched film on the other hand exhibit non-uniform sections widths and non-uniform section heights. Since the same stretching force acts on each section of the film it can be said in general that thinner sections are more prone to stretch than thicker sections. However, since the relationship between force and stretched length is a non-trivial function of many factors exact predictions are difficult to make without resorting to mathematics.

In order to develop a mathematical model of the sideways draw, the concept of strain (stretched length) will be introduced. Strain can be defined as,

$$
\varepsilon_{j}=\frac{\tilde{w}_{j}-w_{j}}{w_{j}}
$$

where $\tilde{w}_{j}$ denote the stretched width of the film in section $j$ and $w_{j}$ denote the un-stretched width of the film in section $j$. Using the above the stretched width can be written as,

$$
\tilde{w}_{j}=w_{j}\left(\varepsilon_{j}+1\right)=\frac{w}{n}
$$

which, since un-stretched width of each section of film is uniform, can be written as,

$$
\tilde{w}_{j}=w_{j}\left(\varepsilon_{j}+1\right)=\frac{w}{n}\left(\varepsilon_{j}+1\right)
$$

where,

$$
w=w_{1}+w_{2}+\cdots+w_{n}
$$

It follows from this that,

$$
\begin{aligned}
\tilde{w}= & \tilde{w}_{1}+\tilde{w}_{2}+\cdots+\tilde{w}_{n} \\
& =\frac{w}{n}\left(\varepsilon_{1}+1\right)+\frac{w}{n}\left(\varepsilon_{2}+1\right)+\cdots+\frac{w}{n}\left(\varepsilon_{n}+1\right)
\end{aligned}
$$

The stress (stretch force) acting on each section of the film can be computed using,

$$
\sigma_{j}=\frac{F}{A_{j}}
$$

where $F$ is the force acting on the $j$ :th section and $A_{j}$ is the cross-sectional area of the $j$ :th section of the film strip.

The stress acting on the $j$ :th section can be related to the strain of the $j$ :th section by a stress-strain relationship Kanai and Campbell [1999]. The stress-strain relationship adopted in this instance is nonlinear function that depends on stretch temperature and the average strain rate,

$$
\sigma_{j}=f\left(\varepsilon_{j}, T_{j}, v_{m}\right)
$$

By combining the above equations a system of nonlinear equations are obtained which can be solved for the force, $F$, and the strains. For this purpose note that the total strain is known and that the stresses are defined by the force, $F$. 


\section{IMPLEMENTATION AND PARAMETER TUNING}

The model described in the previous section has been implemented using Matlab/Simulink. The resulting simulation model encompasses several hundred dynamic and algebraic states, which is reasonably large for a Simulink model in the experience of the authors. Although the initial implementation runs slowly, mainly due to the computationally demanding solution of the algebraic equations associated with the sideways draw, recent developments and experiments of more efficient equation solving routines is expected to solve these issues. The simulation speed when the algebraic equations associated with the sideways draw are replaced by constants is orders of magnitude faster than real time.

The parameters of the model have been tuned using data obtained from DuPont Teijin Films UK Ltd (DTF). This data include equipment specifications as well as operational data. The operational data have been used both for validation and to identify unknown model parameters.

The model will provide a safe off-line platform for developing and testing new monitoring and fault detection tools in the near future.

\section{VALIDATION}

Although the first-principles model described above can predict many different film properties, the intended purpose of the model is to predict the film thicknesses (or the weight per unit of area which is linearly related to the thickness). The validation experiments carried out have therefore mainly focused on the ability of the model to predict this property and ignored other properties such as temperature etc.

Using operational data obtained from DTF, the steady state behaviour of the model has been validated. The validation included mapping the film thicknesses that the model predicts at the die outlet and sideways draw outlet with the real-life operating data. The results showed that the deviations between the model predictions and measurement data remained below $5 \%$, which seems reasonable for a model of this size and complexity.

\section{CONCLUSIONS}

A control and monitoring oriented model of a plastic film manufacturing process is developed in this paper. The model is based in part on fundamental laws of physics and in part on empirical results and have been implemented in Matlab/Simulink and validated using steady state data.

The method of this paper is a novel approach in an area where it is more common to assume an empirical model of the actuators and a random disturbance, or to base diagnostics solely on process data.

\section{REFERENCES}

K.W. Corscadden and S.R. Duncan. Multivariable disturbance modelling for web processes. International Journal of Systems Science, 31(1):97-106, 2000.
S.R. Duncan, J.M. Allwood, W.P. Heath, and K.W. Corscadden. Dynamic modelling of cross-directional actuators: implications for control. IEEE Transactions on Control Systems Technology, 8(3):667-675, 2000.

A.P. Featherstone, J.G. VanAntwerp, and R.D. Braatz. Identification and control of sheet and film processes Springer, London, 2000

T. Kanai and G.A. Campbell, editors. Film Processing Hanser Publishers, Munich, 1999

D.W. Brookes and G.A. Giles. PET Packaging Technology Blackwell, 2002 